Running title: MEMORY FOR EMOTIONAL FACES IN MAJOR DEPRESSION

Word Count: 4251 (excluding the abstract, references and tables)

Memory for emotional faces in major depression following judgement of physical facial characteristics at encoding

\author{
Nathan Ridout \\ Aston University, Birmingham, UK \\ Barbara Dritschel \\ University of St Andrews, UK \\ Keith Matthews and Maureen McVicar \\ University of Dundee, UK \\ Ian C. Reid \\ University of Aberdeen, UK \\ Ronan E. O’ Carroll \\ University of Stirling, UK
}

Address for correspondence:

Dr Nathan Ridout

Clinical and Cognitive Neurosciences Research Group

School of Life and Health Sciences

Aston University

Birmingham, B7 4ET

Tel: (+44 121) 2044162

Fax: (+44 121) 2044090

Email: n.ridout@aston.ac.uk 


\begin{abstract}
The aim of the present study was to establish if patients with major depression (MD) exhibit a memory bias for sad faces, relative to happy and neutral, when the affective element of the faces is not explicitly processed at encoding. To this end, 16 psychiatric outpatients with MD and 18 healthy, never-depressed controls (HC) were presented with a series of emotional faces and were required to identify the gender of the individuals featured in the photographs. Participants were subsequently given a recognition memory test for these faces. At encoding, patients with MD exhibited a non-significant tendency towards slower gender identification (GI) times, relative to HC, for happy faces. However, the GI times of the two groups did not differ for sad or neutral faces. At memory testing, patients with MD did not exhibit the expected memory bias for sad faces. Similarly, HC did not demonstrate enhanced memory for happy faces. Overall, patients with MD were impaired in their memory for the faces relative to the HC. The current findings are consistent with the proposal that moodcongruent memory biases are contingent upon explicit processing of the emotional element of the to-be-remembered material at encoding.
\end{abstract}




\section{Memory for emotional faces in major depression following judgement of physical facial characteristics at encoding}

\section{Introduction}

There has been considerable research effort dedicated to investigating the effect of depression on memory for emotional words, with the key finding being that clinically depressed patients tend to exhibit a memory bias for depression-relevant words relative to positive or neutral (e.g. Bradley, Mogg \& Williams, 1995; Dunbar \& Lishman, 1984; Watkins, Mathews, Williamson \& Fuller, 1992). In contrast there has been relatively little investigation of the effect of depression on memory for nonverbal emotional cues, notably emotional faces. This is surprising given that emotional facial expressions are socially and biologically important (see Darwin, 1872/1965) and thus a negative memory bias for these stimuli could have detrimental effects on the patients' social functioning, and might also act to maintain or even worsen the ongoing depressive episode.

Recently, Ridout, Astell, Reid, Glen \& O’Carroll (2003) conducted a study to establish if the negative memory bias generalises to depressed individuals’ processing of emotional faces and they reported that clinically depressed patients exhibited superior recognition memory for previously viewed sad faces, relative to happy or neutral faces. In contrast, they reported that healthy controls demonstrated enhanced recognition memory for happy faces, relative to neutral or sad. Gilboa-Schechtman, Erhard-Weiss and Jeczemien (2002) also reported a similar memory bias for negative emotional expressions in clinically depressed patients. However, it should be noted that Deveney and Deldin (2004) failed to demonstrate a negative memory bias for emotional faces in a sample of patients with clinical depression, which suggests that 
the negative face memory bias is not always evident in major depression. A key difference between their study and the previous work in this area (Gilboa-Schechtman et al, 2002; Ridout et al, 2003) was the participants were not required to explicitly process the emotional content of the faces during the encoding phase. This difference could plausibly explain the lack of memory bias in their study, as, according to the influential Interacting Cognitive Subsystems (ICS) model (Teasdale \& Barnard, 1993), mood congruent memory is dependent upon explicit encoding of the emotional element of the to-be-remembered stimuli.

The encoding phase of the study conducted by Ridout et al (2003) involved the participants identifying the emotion that was being portrayed by the individual featured in each photograph. This task would have required explicit processing of the emotional element of each stimulus. It is theoretically, and potentially clinically, important to establish if the memory bias for emotional faces would still be observed if the encoding phase did not involve direct processing of the emotional valence. Therefore, in order to address this question a partial replication of experiment reported in Ridout et al (2003) was conducted using an encoding task (gender identification) that did not require explicit processing of the emotional element of the faces.

Although the proposed study will make use of an explicit memory task, i.e. the participants will be informed that their memory will be tested for the faces, the literature on implicit memory biases in depression is still of relevance to the current study, given that any observed negative memory bias would presumably be a consequence of implicit processing. The most recent evidence (see review of Barry, Naus \& Rehm, 2004) demonstrates that depressed individuals do exhibit negatively biased implicit processing, but only in circumstances where there is congruence 
between the type of processing (perceptual or conceptual) required at encoding and retrieval.

Ferré (2003) conducted an experiment that is particularly relevant to the current study. Healthy participants were presented with positive, negative and neutral words under two encoding conditions (semantic and physical) and were subsequently asked to recall the words. The author reported that, following semantic encoding, participants exhibited superior recall of emotional words (positive \& negative) relative to neutral. However, following physical encoding the memory advantage was only evident for positive words. The finding of a positive memory bias even when the 'emotional charge' of the words was not explicitly processed at encoding is important for the current study, as it shows that implicit processing of emotion can influence explicit memory retrieval. Given these findings it is necessary to consider the possible influence of implicit emotional processing on memory for the faces.

Another body of evidence that is potentially relevant to the present study concerns the tendency of depressed individuals to selectively attend to negative material in their environment. For example, studies that have utilised emotional variants of the Stroop colour-naming task (e.g. Gotlib \& Cane, 1987; Nunn, Mathews \& Trower, 1997; Segal et al, 1995) have tended to demonstrate that depressed individuals are slower to colour-name depression-relevant words than positive or neutral words (usually interpreted as evidence of attention capture by the negative material). It has also been reported that patients with depression exhibit biased attentional processing of emotional faces (e.g. Gotlib, Krasnoperova, Yue, \& Joormann, 2004). These findings have implications for the present study, as such biases could influence the processing of the faces and hence the patients' memory for them. 
If Teasdale and Barnard's ICS model is correct then there should be no evidence of a depression-related memory bias in the present study. However, the findings of attentional \& implicit memory biases in depression, and the findings of Ferré (2003), would suggest that patients with MD might exhibit superior memory for sad faces relative to neutral or happy, in line with previous studies (GilboaSchechtman et al, 2002; Ridout et al, 2003). Furthermore, based on Ferré (2003), it is expected that healthy controls will exhibit enhanced memory for happy faces relative to sad and neutral.

\section{METHOD}

\section{Participants}

Sixteen psychiatric outpatients (5 male \& 11 female) with an ICD-10 diagnosis of major depression and 18 healthy, never-depressed participants (4 male \& 14 female) took part in the present study. The two groups were matched in terms of their age, sex, educational background (years of full-time education completed), and pre-morbid intellectual ability (estimated from the participants' scores on the National Adult Reading Task; NART, Nelson \& Williamson, 1991). The patients were recruited from the University of Dundee Affective Disorders Clinic (ADC) at Ninewells Hospital and Medical School in Dundee. The patients’ diagnosis was established by one of the psychiatrists at the ADC, who also rated the severity of the patients' depression using the 17-item Hamilton Rating Scale for Depression (HRSD; Hamilton, 1961). The mean HRSD score for the patients in the current study was 20 (with a range of $14-24$ ). In addition to an ICD-10 diagnosis of major depression (without psychotic or bipolar symptoms) the inclusion criteria for the present study were a depression severity (HRSD) score of 14 or above and aged between 18 and 65 . 
The exclusion criteria included a primary diagnosis other than major depression (e.g. social phobia), recent treatment (within 6 months prior to testing) with electroconvulsive therapy (ECT), a history of head trauma involving prolonged loss of consciousness and subsequent hospitalisation, suspected alcohol or substance dependence, and the presence of a physical condition, or treatment with a medication, considered likely to adversely affect cognitive performance (e.g. multiple sclerosis; benzodiazepines). It should be noted that all of the patients with MD were being treated with antidepressant medication at the time of testing, with 11 being treated with selective serotonin re-uptake inhibitors (SSRIs), 3 with tricyclic antidepressants and 2 with monoamine oxidase inhibitors (MAOIs). The inclusion criteria for the healthy controls were; an absence of current or previous depression (established using a structured screening questionnaire) and meeting the matching criteria for the clinical sample. Exclusion criteria were the same as for the depressed sample. This study was approved by the Tayside Committee for Medical Research Ethics.

\section{Materials and measures}

Emotional faces: The facial stimuli utilised in the present study consisted of 50 grey scale photographic images of different individuals portraying emotional facial expressions. Thirty of these individuals (15 male, 15 female) portrayed both happiness and sadness. The remaining twenty (10 male, 10 female) portrayed a neutral expression. Two sets (A \& B) of 30 stimuli were constructed for the encoding phase. Each set featured 10 happy, 10 neutral and 10 sad faces and consisted of 15 male and 15 female faces. Individuals portraying happiness in set A portrayed sadness in set B and vice versa. Different individuals portrayed the neutral expressions in sets A \& B. These procedures were used to control for the influence of facial 
distinctiveness upon subsequent recognition memory. Reanalysis of the emotion identification accuracy data from a previous study (Ridout et al, 2003) revealed that the two sets of faces did not differ in terms of perceived happiness (Set $A=93.3 \%$, $\mathrm{SE}=4.2$; Set $\mathrm{B}=97.3 \%, \mathrm{SE}=1.8$ ), sadness (Set $\mathrm{A}=82.7 \%$, $\mathrm{SE}=4.3$; Set $\mathrm{B}=85.3 \%$, $\mathrm{SE}=3.1$ ) or neutral affect (Set $\mathrm{A}=60.1 \%, \mathrm{SE}=3.9$; Set $\mathrm{B}=60.1 \%, \mathrm{SE}=2.8)$; $\mathrm{t}(28)=0.9$, $\mathrm{p}>0.05, \mathrm{t}(28)=0.5, \mathrm{p}>0.05$ and $\mathrm{t}(28)=0, \mathrm{p}>0.05$. Two sets of stimuli $\left(\mathrm{A}^{\mathrm{m}} \& \mathrm{~B}^{\mathrm{m}}\right)$ were also compiled for the recognition memory test. Set $\mathrm{A}^{\mathrm{m}}$ featured 30 familiar faces (encoding set A) plus 20 novel faces (5 happy, 5 sad \& 10 neutral ${ }^{1}$ ). Set $\mathrm{B}^{\mathrm{m}}$ consisted of 30 familiar (encoding set B) and 20 novel faces. As with the faces at encoding, individuals portraying happiness in Set $\mathrm{A}^{\mathrm{m}}$ portrayed sadness in Set $\mathrm{B}^{\mathrm{m}}$ and vice versa.

General cognitive assessment: In order to establish if the two groups differed in terms of the time taken to detect and respond to stimuli, a simple reaction time task was included. This task involved participants monitoring a computer screen for a period of around 3 minutes, during which time a simple stimulus (solid red circle) would appear at random intervals (500, 750, 1000, 1250 \& 1500 milliseconds). The participants were invited to press a key as quickly as they could once the stimulus had appeared. The computer recorded the time taken to respond to each stimulus. Overall, the task consisted of 60 stimulus presentations (plus 10 practice trials). The Digit Symbol Substitution Task (DSST) and Digit Span Task (Wechsler, 1981) were included as distracter tasks during the five-minute delay period between the encoding and recognition memory testing. The National Adult Reading Test (NART; Nelson \& Williamson, 1991) was included in the current study in order to provide an estimate of

\footnotetext{
${ }^{1}$ The novel neutral faces in set $\mathrm{A}^{\mathrm{m}}$ were drawn from encoding set $\mathrm{B}$, whereas the novel neutral faces in set $\mathrm{B}^{\mathrm{m}}$ were drawn from encoding set $\mathrm{A}$.
} 
the participants’ pre-morbid intellectual ability. This task has been shown to provide a reasonable estimate of full IQ score that is independent of the severity of participants’ depression (Crawford et al, 1987).

Measurement of self-rated mood: The 21-item Beck Depression Inventory (BDI; Beck et al, 1961) was utilised in the present study to provide and index of selfrated depression in the patients, and to confirm the absence of significant depressive symptoms in the healthy controls. The 14-item Hospital Anxiety and Depression Scale (HADS; Zigmond \& Snaith, 1983) was used primarily to provide an index of co-morbid anxiety in the patients with MD and also to screen for the presence of significant anxiety symptoms in the controls.

\section{Procedure}

The participants were presented with one of the sets (A or B) of 30 faces and were asked to indicate, via a key press, whether the person featured in each photograph was a male or female. The faces remained on screen until the participants had made their decision. However, the participants were urged to respond as quickly and accurately as they could. Following completion of the gender identification task, there was a five-minute filled delay, during which time the participants were asked to complete the DSST and digit span tasks (Wechsler, 1981). Participants were subsequently given a recognition memory test for the faces they had viewed during the gender decision phase. During memory testing participants were presented with the appropriate set of faces (i.e. if they had viewed set A at encoding they would be presented with set Am at memory testing) and were required to indicate, via a key press, whether they recognised the faces from the gender identification phase. In line 
with the encoding phase, the faces remained on screen until the participants responded and participants were urged to make their judgements as quickly and accurately as possible. Once all of the faces had been viewed and judged, the participants were asked to complete the simple reaction time task and the NART. Finally, they were asked to complete the self-report measures of mood (BDI and HADS).

\section{RESULTS}

\section{Participant characteristics}

Analysis of the participant characteristics revealed that the patients with major depression (MD) did not differ from the HC in terms of their age (MD Mean= 43.7 years, Standard Deviation=11.3, HC Mean=39.3 years, SD=8.8), sex ratio (M:F; $\mathrm{MD}=5: 11, \mathrm{HC}=4: 14)$, educational background $(\mathrm{MD}=12.6$ years, $\mathrm{SD}=0.9, \mathrm{HC}=13.4$ years, $\mathrm{SD}=0.5)$ and estimated pre-morbid intelligence $(\mathrm{MD}=113.6, \mathrm{SD}=1.1$, $\mathrm{HC}=114.1, \quad \mathrm{SD}=0.8) ; \quad \mathrm{F}(1, \quad 32)=1.6, \quad \mathrm{p}>0.05, \quad \chi^{2}(1)=0.7, \quad \mathrm{p}>0.05, \quad \mathrm{~F}<1 \quad$ and $\mathrm{F}<1$ respectively. However, as expected, MD rated themselves as significantly more depressed than did the $\mathrm{HC}$ on both the $\mathrm{BDI}(\mathrm{MD}=31.8, \mathrm{SD}=1.8, \mathrm{HC}=2.6, \mathrm{SD}=0.4)$ and the HADS depression subscale ( $\mathrm{MD}=14.6, \mathrm{SD}=0.7, \mathrm{HC}=0.8, \mathrm{SD}=0.2) ; \mathrm{F}(1$, 32)=277.5, $\mathrm{p}<0.001$ and $\mathrm{F}(1,32)=418.6, \mathrm{p}<0.001$ respectively. $\mathrm{MD}$ also rated themselves as significantly more anxious on the HADS anxiety subscale (Mean=14.1, $\mathrm{SD}=0.5)$ than did the $\mathrm{HC}(\mathrm{M}=3.4, \mathrm{SD}=0.7), \mathrm{F}(1,26)=147.4, \mathrm{p}<0.001$.

\section{General cognitive assessment}

MD made significantly fewer symbol substitutions on the DSST (Mean=42.3, Standard Error=2.3) than did the HC $(\mathrm{M}=58.0, \mathrm{SE}=2.1) ; \mathrm{F}(1,32)=25.5, \mathrm{p}<0.001$. They also remembered fewer digit spans $(M=15, S E=0.6)$ than did the HC $(M=16.9$, 
$\mathrm{SE}=0.7)$, a difference that approached conventional significance, $\mathrm{F}(1,32)=3.9$, $\mathrm{p}=0.056$. Furthermore, the MD were significantly slower on the simple reaction time task $(M=355.4 \mathrm{~ms}, \mathrm{SE}=23.7)$ than were the $\mathrm{HC}(\mathrm{M}=324 \mathrm{~ms}, \mathrm{SE}=13.6) ; \mathrm{F}(1,32)=5.5$, $\mathrm{p}<0.05$.

\section{Gender identification accuracy}

Inspection of the gender identification accuracy scores revealed that the performance of the participants in both groups was at ceiling, for happy (MD=100\%, $\mathrm{HC}=99.5)$, neutral $(\mathrm{MD}=96.6 \%, \mathrm{HC}=98.1 \%)$ and sad faces $(\mathrm{MD}=99.5 \%, \mathrm{HC}=99.5 \%)$.

\section{Gender identification times}

As noted above, the psychomotor speed of the patients with MD is significantly slower than that of the HC (indexed by simple reaction time). As this difference could confound the interpretation of the participants' gender identification times, psychomotor speed was controlled for statistically by entering SRT into the analysis as a covariate. Although statistical analysis revealed no significant difference between the groups in terms of age, inspection of the data revealed a difference of nearly four years. Therefore, in order to ensure this difference in age did not influence the participants' gender identification times, age was also entered as a covariate into the analysis. A 2(Group; depressed vs. controls) x 3(Emotion; happy vs. neutral vs. sad) repeated measures ANCOVA was used to analyse the participants’ gender identification times (adjusted means are illustrated in Figure 1). The results of this analysis revealed a significant main effect of simple reaction time; $F(1,30)=10.7$, 
$\mathrm{p}<0.01$. There was also a significant Group $x$ Emotion interaction; $F(2,60)=3.7$, $\mathrm{p}<0.05$. However, no other main effects or interactions were significant.

In order to elucidate the nature of the Group x Emotion interaction separate one-way repeated measures ANCOVA were conducted for each group (alpha level adjusted for multiple comparisons using the Bonferroni correction method) and revealed that the gender identification times of the patients with MD and healthy controls did not differ significantly as a function of type of emotional expression; $\mathrm{F}(2$, $26)=2.7, \mathrm{p}>0.05$ and $\mathrm{F}(2,30)=1.6, \mathrm{p}>0.05$ respectively. Inspection of figure 1 revealed that there was no apparent difference between the two groups in terms of their gender identification times for neutral and sad expressions. However, the patients with MD appeared to be markedly slower (adjusted mean=807.1 milliseconds, standard error=36.6) than the controls (adjusted $\mathrm{M}=705.1 \mathrm{~ms}, \mathrm{SE}=33.7$ ) in identifying the gender of happy faces. In order to assess the significance of this difference, a univariate ANCOVA was conducted with gender identification times for happy faces entered as the dependent variable, group entered as the independent variable and age \& simple reaction time entered as covariates. The results of this analysis revealed that this difference failed to reach conventional significance once the influence of age and simple reaction time were controlled for; $F(1,30)=3.77$, $\mathrm{p}=0.06$.

Correlational analysis - gender identification times

Partial correlations (controlling for age and simple reaction time) were utilised to investigate the relationships between the participants' self-rated depression \& anxiety and their gender identification times for happy faces. These analyses revealed 
that both depression and anxiety scores were significantly positively related to gender identification times for happy faces; $\mathrm{r}(30)=0.37, \mathrm{p}<0.05$ and $\mathrm{r}(30)=0.4, \mathrm{p}<0.05$ respectively. It should be noted that there was also a highly significant positive correlation between the participants' depression and anxiety scores; $r(30)=0.9$, $\mathrm{p}<0.001$. Further analyses were conducted controlling for depression and anxiety respectively. When depression severity was controlled for, the relationship between anxiety and gender identification times for happy faces was considerably weaker and no longer significant; $r(29)=0.17, p>0.05$. When anxiety was controlled for, there was no longer evidence of a relationship between depression and gender identification time; $r(29)=0.03, \mathrm{p}>0.05$.

\section{Recognition memory for emotional faces}

The percentage of each type of emotional face correctly recognised by the participants in the two groups during memory testing (illustrated in figure 2) was analysed using a 2(Group; depressed vs. controls) x 3(Emotion; happy vs. neutral vs. sad) mixed ANCOVA, with age entered as a covariate. Results revealed that patients with MD exhibited significantly poorer memory for the faces (Mean=69.5\%, SE=1.7) relative to the controls $(\mathrm{M}=79.2 \%, \mathrm{SE}=1.6) ; \mathrm{F}(1,31)=16.3, \mathrm{p}<0.001$. However, there were no significant main effects of emotion or age on memory for the faces; $F(2$, $62)=2.8, \mathrm{p}>0.05$ and $\mathrm{F}(1,31)=3.4, \mathrm{p}>0.05$ respectively. Moreover, there was no significant Group x Age or Group x Emotion interactions; F(2, 62)=2.8, p>0.05 and $\mathrm{F}(2,62)=2.9, \mathrm{p}>0.05$ respectively. 
Correlational analysis - recognition memory phase

Partial correlations (controlling for age) were utilised to establish if the observed group difference in recognition memory for the faces was associated with the degree of depression and/or anxiety. These analyses revealed that the percentage of faces correctly recognised during memory testing was significantly negatively related to both depression and anxiety; $r(31)=-0.54, p<0.01$ and $r(31)=-0.51, p<0.01$ respectively. As noted previously, there was a strong positive correlation between depression and anxiety scores, $r(31)=0.9, \mathrm{p}<0.001$. Further analyses were conducted controlling for depression and anxiety in turn. When depression was controlled for, there was no longer evidence of a relationship between anxiety and recognition memory for the faces; $r(31)=-0.03, \mathrm{p}>0.05$. When anxiety was controlled for, the relationship between depression and memory for the faces was considerably weaker and no longer significant, $r(31)=-0.22, \mathrm{p}>0.05$.

\section{DISCUSSION}

The aim of the present study was to establish if patients with major depression (MD) would exhibit a memory bias for sad faces, relative to happy and neutral, when the affective element of the faces was not explicitly processed at encoding. Given the findings of attentional (Gotlib et al, 2004) and implicit memory biases (Barry et al, 2004) in depression and the evidence of emotional memory biases in the absence of explicit emotional processing (Ferré, 2003) it was predicted that the patients with major depression (MD) might exhibit enhanced memory for the sad faces, in line with previous studies (Gilboa-Schechtman et al, 2002; Ridout et al, 2003). However, contrary to this prediction, patients with MD did not exhibit enhanced memory for the sad faces, as they demonstrated equivalent memory of all three types of emotional 
face. In line with Ferré (2003), it was expected that the healthy controls would demonstrate superior memory for happy faces relative to sad and neutral. However, contrary to this proposition, the controls demonstrated equivalent memory for all three types of emotional face, in line with the pattern observed for the patients with MD.

These findings suggest that implicit processing of affective content does not influence explicit memory for emotional faces. Furthermore, the lack of moodcongruent biases in the present study is inconsistent with the findings for affective verbal material reported in Ferré (2003), which suggests that the two forms of stimuli are processed in different ways. However, it is possible that conditions in the present study were not conducive to inducing implicit memory biases. For example, it is unclear whether there was complete congruence between the type of processing required at encoding and retrieval, which is has been identified as vital for implicit memory bias (Barry et al, 2004). Making a gender decision would presumably have relied largely on perceptual processing. However, although it can be assumed that the participants would also utilise perceptual mechanisms to make their recognition judgements, they were not constrained in how they made their decisions and so could plausibly have used other more affective methods, i.e. a feeling of familiarity. This could be addressed in the future by directing the participants to use particular retrieval strategies and/or taking a measure of the memory 'experience' i.e. remember/ know judgements (Gardiner, Ramponi \& Richardson-Klavehn, 1998). On the basis of the current findings it has to be concluded there is no evidence that implicit processes influenced the memory for emotional faces. However, it is not possible to make strong claims based on a single study.

The lack of mood congruent memory biases reported in the present study is consistent with the ICS model of Teasdale and Barnard (1993), as they proposed that 
mood congruent memory biases will only be evident in circumstances that involve explicit processing of the affective element of the to-be-remembered material at encoding. In the present study, the encoding task (gender identification) did not require explicit processing of the emotional content of the faces; hence this information would not have been encoded and therefore arguably would not have influenced the participants' memory for the faces.

The finding that patients with MD correctly recognised fewer faces than the HC at memory testing is consistent with previous studies reporting impaired recognition memory for faces in depressed participants (e.g. Brown et al, 1994; Palmer et al, 1996).

The finding that MD were slower to identify the gender of the happy faces, suggests that the emotional element of these faces was being processed and that this processing interfered with the patients' performance of the task in hand (gender identification). This finding is inconsistent with previous studies that have utilised emotional versions of the Stroop colour-naming task to investigate attentional processing of emotional stimuli in patients with MD (e.g. Gotlib \& Cane, 1987; Nunn, Mathews \& Trower, 1997; Segal et al, 1995), as they reported interference from negative as opposed to positive stimuli. However, it should be noted that the observed difference in the present study failed to reach conventional significance and thus would require replication with a larger sample before any implications could be considered.

Limitations of the current study include the relatively small sample sizes and relatively small number of stimuli presented during encoding. Nevertheless, it should be noted that the same sample size and number of stimuli was sufficient to demonstrate marked MCM biases in the previous study (Ridout et al, 2003). Another 
limitation of the present study concerns the lack of a controlled cross-over design. It would have been preferable to have tested the participants' memory following two encoding tasks, one that encouraged explicit processing of emotion (e.g. emotion identification) and one that did not (gender identification). If participants exhibited a memory bias following emotion identification but not after gender identification, this would have provided stronger evidence of the necessity of explicit emotion processing. Nevertheless, the current study still provides important data against the backdrop of previous work in the area (Gilboa-Schechtman et al, 2002; Ridout et al, 2003). A further issue that requires note is that all patients in the depressed sample were being treated with antidepressant medication at the time of testing. It is plausible that the observed impairments in the patients with MD' memory for the sad and neutral faces relates to drug induced deficits in memory function. However, it should be noted that the patients in the depressed sample of our previous study (Ridout et al, 2003) were also treated with the same regimen of antidepressants. Furthermore, evidence suggests that modern anti-depressants (notably the Selective Serotonin Reuptake Inhibitors, SSRIs) are associated with relatively little impairment of memory function in patients with MD, and may even result in an improvement on pre-treatment performance (Brambilla et al, 2005; Levkovitz et al, 2002; Oxman, 1996). A final limitation concerns the presence of co-morbid anxiety in the patients with MD. There is considerable evidence that anxiety, in addition to depression, is associated with biased processing of emotional stimuli (see Williams, Watts, MacLeod \& Mathews, 1997). Therefore the significant difference in anxiety between the groups could have influenced the results in the present study. Inspection of the results did reveal that gender identification times for happy faces and the percentage of faces correctly recognised at memory testing were both significantly related to 
severity of anxiety as well as depression. This is not surprising given that depression and anxiety scores were themselves extremely highly correlated. The presence of comorbid anxiety makes the current results harder to interpret. Nevertheless, the partial correlations suggest that anxiety exerted a greater influence on the gender identification times for happy faces. Conversely, depression appears to be more important in explaining the observed deficit in memory for the faces. It is important in the future to try and investigate the memory function of individuals with 'pure' depression in the absence of anxiety. However, this may prove difficult given that there is a high co-morbidity rate (typically greater than 50\%) between depression and anxiety (Mineka, Watson \& Clark, 1998).

In summary, the present study reported that, following a non-emotional encoding task (gender identification), patients with MD exhibited no evidence of a memory bias for sad faces. Similarly, there was no evidence of a happy face bias in the healthy controls. Overall, the patients with MD exhibited impaired memory for the faces relative to the controls. The failure to produce mood congruent memory (MCM) biases in the present study is consistent with the predictions of Teasdale \& Barnard’s ICS model (1993), as they proposed that MCM biases are dependent upon the affective content of the to-be-remembered stimuli being explicitly processed at encoding. 


\section{References}

Barry, E. S., Naus, M. J., \& Rehm, L. P. (2004) Depression and implicit memory: Understanding mood congruent memory bias. Cognitive Therapy and Research, 28(3), 387 - 414

Beck, A. T., Ward, C. H., Mendelson, C. H., Mock, J., \& Erlbaugh, J. (1961). An inventory for measuring depression. Archives of General Psychiatry, 4, 561 571.

Bradley, B. P., Mogg, K., \& Williams, R. (1995). Implicit and explicit memory for emotion-congruent information in clinical depression and anxiety. Behavior Research and Therapy, 33(7), 755-770.

Brambilla, P., Cipriani, A., Hotopf, M., \& Barbui, C. (2005) Side effect profile of Fluoxetine in comparison with other SSRIs, Tricyclic and newer antidepressants: A meta-analysis of clinical trial data. Pharmacopsychiatry, 38, $69-77$

Brown, R. G., Scott, L. C., Bench, C. J., \& Dolan, R. J. (1994). Cognitive function in depression: its relationship to the presence and severity of intellectual decline. Psychological Medicine, 24(4), 829-847.

Crawford, J. R., Besson, J. A. O., Parker, D. M., Sutherland, K. M., \& Keen, P. L. (1987). Estimation of premorbid intellectual status in depression. British Journal of Clinical Psychology, 26, 313 - 314.

Darwin C. (1872/1965) The Expression of the Emotions in Man and Animals Chicago: University of Chicago

Deveney, C. M., \& Deldin, P. J. (2004). Memory of faces: a slow wave ERP study of major depression. Emotion, 4(3), 295-304 
Dunbar, G. C., \& Lishman, W. A. (1984). Depression, recognition-memory and hedonic tone a signal detection analysis. British Journal of Psychiatry, 144, 376-382.

Ferré, P. (2003) Effects of level of processing on memory for affectively valenced words. Cognition and Emotion, 17(6), 859 - 880

Gardiner, J. M., Ramponi, C. \& Richardson-Klavehn, A. (1998) Experiences of remembering, knowing and guessing. Consciousness and Cognition, 7, 1-26

Gilboa-Schechtman, E., Erhard-Weiss, D., \& Jeczemien, P. (2002) Interpersonal deficits meet cognitive biases: memory for facial expressions in depressed and anxious men and women. Psychiatry Research, 113(3), 279 - 293

Gotlib, I. H., \& Cane, D. B. (1987). Construct accessibility and clinical depression: a longitudinal investigation. Journal of Abnormal Psychology, 96, 199 - 204.

Gotlib, I. H., Krasnoperova, E., Yue, D. N., \& Joormann, J. (2004). Attentional biases for negative interpersonal stimuli in clinical depression. J Abnorm Psychol, 113(1), 121-135.

Hamilton, M. (1960). A rating scale for depression. Journal of Neurology, Neurosurgery and Psychiatry, 23, 56-62.

Levkovitz, Y., Caftori, R., Avital, A., \& Richter-Levin, G. (2002) The SSRI drug Fluoxetine, but not the noradrenergic tricyclic drug Desipramine improves memory performance during acute major depression. Brain Research Bulletin 58(4), $345-350$

Mineka, S., Watson, D., \& Clark, L. A. (1998) Comorbidity of anxiety and unipolar mood disorders. Annu Rev Psychol, 49, 377-412

Nelson, H. E., \& Williamson, J. (1991). National Adult Reading Test (NART) Test Manual (second ed.). Windsor: NFER - NELSON. 
Nunn, J. D., Mathews, A., \& Trower, P. (1997). Selective processing of concernrelated information in depression. British Journal of Clinical Psychology, 36(Pt 4), 489-503.

Oxman, T. E. (1996) Antidepressants and cognitive impairment in the elderly. Journal of Clinical Psychiatry, 57, suppl. 5 38-44

Palmer, B. W., Boone, K. B., Lesser, I. M., Wohl, M. A., Berman, N., \& Miller, B. L. (1996). Neuropsychological deficits among older patients with MD with predominantly psychological or vegetative symptoms. Journal of Affective Disorders, 41(1), 17-24

Ridout, N., Astell, A. J., Reid, I. C., Glen, T., \& O'Carroll, R. E. (2003). Memory bias for emotional facial expressions in major depression. Cognition and Emotion, 17(1), $101-122$.

Segal, Z. V., Gemar, M., Truchon, C., Guirguis, M., \& Horowitz, L. M. (1995). A priming methodology for studying self-representation in major depressive disorder. Journal of Abnormal Psychology, 104(1), 205-213.

Teasdale, J. D., \& Barnard, P. J. (1993). Affect, Cognition and Change: Remodelling depressive thought. Hove: Lawrence Erlbaum Associates

Watkins, P. C., Mathews, A., Williamson, D. A., \& Fuller, R. D. (1992). Moodcongruent memory in depression: Emotional priming or elaboration? Journal of Abnormal Psychology, 101(3), 581-586.

Wechsler, D. (1981). Wechsler adult intelligence scale-Revised. New York: Psychological Corporation.

Williams, J.M.G., Watts, F.N., MacLeod, C. \& Mathews, A. (1997) Cognitive psychology and emotional disorders ( $2^{\text {nd }}$ ed). Chichester, UK: Wiley. 
Zigmond, A. S., \& Snaith, R. P. (1983). The hospital anxiety and depression scale. Acta Psychiatr Scand, 67(6), 361-370. 


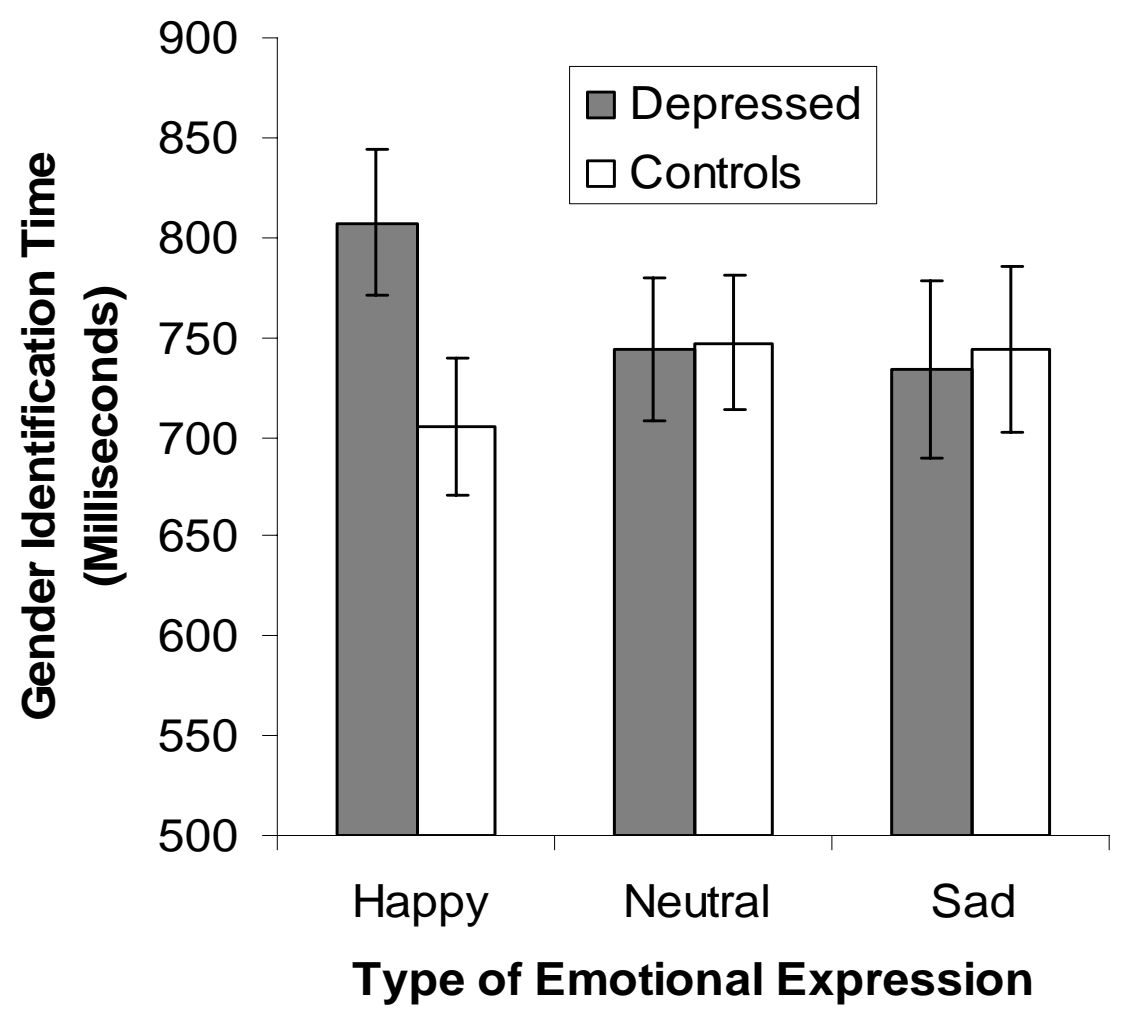

\section{Figure 1}

Gender identification times (adjusted) for the patients with MD and healthy controls, as a function of featured emotional expression (error bars show \pm one standard error of the mean) 


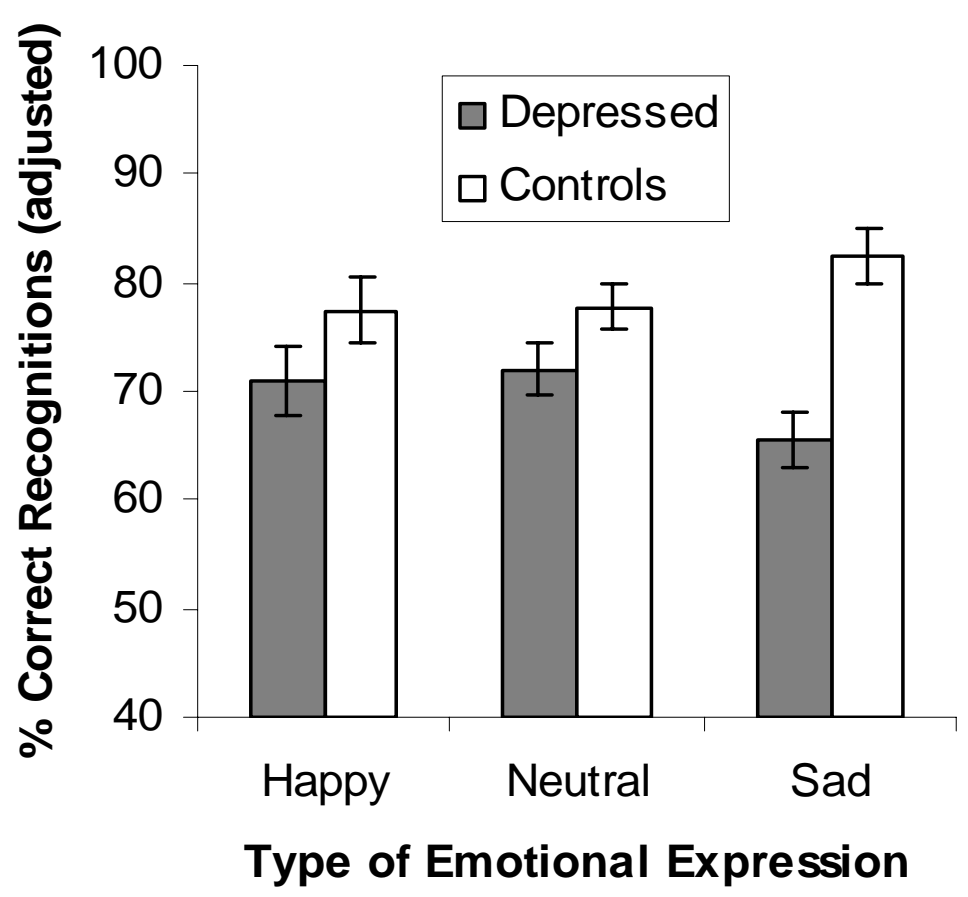

Figure 2: Percentage (adjusted) of each type of facial expression correctly recognised by the participants during memory testing (error bars show \pm one standard error of the mean) 